\title{
RANDOM COMPACT SETS RELATED TO THE KAKEYA PROBLEM
}

\author{
RALPH ALEXANDER
}

ABSTRACT. A $B$-set is defined to be a compact planar set of zero measure which contains a translate of any line segment lying in a disk of diameter one. A construction is given which associates a unique compact planar set with each sequence in a closed interval, and it is shown that for almost all such sequences a $B$ oset is obtained. The construction depends on the measure properties of certain perfect linear sets. Several related problems of a subtler nature are also considered.

1. Introduction. Long ago Besicovitch [1] gave his famous example of a compact planar set of measure zero which contains a translate of every line segment lying in a disk of diameter one. For convenience we will call such a set a $B$-set. Although the original construction of Besicovitch was rather complicated, there have been a number of elegant simplifications, especially for the construction of sets of measure $\epsilon$ containing the required line segments. The idea of Schoenberg discussed in [3] is particularly successful.

In this article we give a simple probabilistic method for generating a large family of $B$-sets. We only need elementary results about the measure of certain linear sets, and a rudimentary knowledge of random sequences.

There are a number of subtle questions which do arise, however. We are able to deal with several of these by appealing to a deep theorem of Besicovitch [2] concerning planar sets of finite Carathéodory length.

2. The measure of certain linear sets. Let $a, b$ and $x_{1}$ lie in the interval $[0,2 / 3]$. Consider the three closed intervals $[a, a+1 / 3],[b, b+$ $1 / 3]$, and $\left[x_{1}, x_{1}+1 / 3\right]$. Let $K\left(x_{1}\right)$ denote this collection of intervals, and let $T\left(x_{1}\right)$ denote their union.

We will form the collection $K\left(x_{1}, x_{2}\right)$, consisting of nine intervals of length $1 / 9$, as follows: For each member $[y, y+1 / 3]$ in $K\left(x_{1}\right)$, there is precisely one affine transformation $\tau$ of the line such that $\tau(0)=y$ and $\tau(1)=y+1 / 3$. The images under $\tau$ of the three intervals in $K\left(x_{2}\right)$ will be three intervals of length $1 / 9$ lying in $[y, y+1 / 3]$. Applying this construction to each of the three intervals in $K\left(x_{1}\right)$ yields the nine intervals in $K\left(x_{1}, x_{2}\right)$. 
Inductively, if $a, b, x_{1}, \ldots, x_{n}$ are numbers in $[0,2 / 3], K\left(x_{1}, \ldots, x_{n}\right)$ will consist of $3^{n}$ intervals of length $3^{-n}$. For each interval $\left[y, y+3^{-n^{+1}}\right]$ in $K\left(x_{1}, \ldots, x_{n-1}\right)$, there will be an affine transformation $\tau$ such that $\tau(0)=y$ and $\tau(1)=y+3^{-n^{+1}}$. The images of the three intervals in $K\left(x_{n}\right)$ will be three intervals in $\left[y, y+3^{-n+1}\right]$. Thus we obtain the $3^{n}$ intervals in $K\left(x_{1}, \ldots, x_{n}\right) . T\left(x_{1}, \ldots, x_{n}\right)$ will denote the union of these intervals, and $l\left(x_{1}, \ldots, x_{n}\right)$ will denote the linear measure of this union. Clearly, $T\left(x_{1}, \ldots, x_{n}\right) \subset T\left(x_{1}, \ldots, x_{n-1}\right)$ so that $l\left(x_{1}, \ldots, x_{n}\right) \leq l\left(x_{1}, \ldots, x_{n-1}\right)$.

If $a, b, x_{1}, x_{2}, \ldots$ is a sequence in $[0,2 / 3]$, we define $T\left(x_{1}, x_{2}, \ldots\right)$ to be $\bigcap_{n} T\left(x_{1}, \ldots, x_{n}\right)$ and $l\left(x_{1}, x_{2}, \ldots\right)$ to be the measure of $T\left(x_{1}, x_{2}, \ldots\right)$. We note that when $a=0$ and $b=2 / 3, T(1 / 3,1 / 3, \ldots)$ is the entire unit interval, while $T(0,0, \ldots)$ is the usual Cantor set. We also observe that if $x_{1}, x_{2}=\cdots=x_{n}=a$, then $l\left(x_{1}, \ldots, x_{n}\right) \leq(2 / 3)^{n}$, since $T\left(x_{1}, \ldots, x_{n}\right)$ can be expressed as the union of $2^{n}$ intervals of length $3^{-n}$.

Lemma 1. Let $a, b, x_{1}, x_{2}, \ldots$ be a sequence in $[0,2 / 3]$. Then for any positive integers $m, n$ we have the inequalities

$$
l\left(x_{1}, x_{2}, \ldots\right)<l\left(x_{m+1}, x_{m+2}, \ldots\right) \leq l\left(x_{m+1}, \ldots, x_{m+n}\right) .
$$

Proof. It is clear from the definitions that $l\left(x_{m+1}, x_{m+2}, \ldots\right) \leq$ $l\left(x_{m+1}, \ldots, x_{m+n}\right)$. We note that $T\left(x_{1}, x_{2}, \ldots\right)$ is the union of $3^{m}$ similar images of $T\left(x_{m+1}, x_{m+2}, \ldots\right)$ and the ratio of similarity is $3^{-m}$ for each image. The inequality $l\left(x_{1}, x_{2}, \ldots\right) \leq l\left(x_{m+1}, x_{m+2}, \ldots\right)$ follows at once.

Lemma ?. Let $a$ and $b$ be numbers in the interval $[0,2 / 3]$. The function $l\left(x_{1}, \ldots, x_{n}\right)$ from $[0,2 / 3]^{n}$ to the interval $[0,1]$ is continuous.

The lemma is obviously true and we omit a proof. Questions concerning the modulus of continuity of $l$ seem difficult, however.

Proposition 1. Almost all sequences $x_{1}, x_{2}, \ldots$ in $[0,2 / 3]$ have the property that given any $a$ and $b$ in $[0,2 / 3]$, then $l\left(x_{1}, x_{2}, \ldots\right)=0$.

Proof. It follows from the classical results of E. Borel and others that given any positive number $\delta$ and positive integer $n$, almost all sequences in $[0,2 / 3]$ have the property that for any number $a$ in $[0,2 / 3]$, the inequality $\left|x_{i}-a\right|<\delta$ will be satisfied for at least $n$ successive values of $i$. (See [4, Problem 5, p. 1971.)

Now by Lemma 2 there is a $\delta$ such that if $\left|x_{i}-a\right|<\delta$ for $i=m+1$, $\ldots, m+n$, then $l\left(x_{m+1}, \ldots, x_{m+n}\right)<(2 / 3)^{n}+\epsilon$. However, it follows from inequality (1) that $l\left(x_{1}, x_{2}, \ldots\right)<(2 / 3)^{n}+\epsilon$. Since $n$ can be arbitrarily large, $l\left(x_{1}, x, \ldots\right)=0$. This concludes the proof.

We remark that "good" sequences are easy to find. For example, we could choose $x_{i}$ to be the fractional part of $(2 / 3)\left(\Sigma_{j=1}^{i} 1^{\prime} j\right)$. The method of 
proof for Proposition 1 makes it clear that for any $a$ and $b$ in $[0,2 / 3]$, $l\left(x_{1}, x_{2}, \ldots\right)=0$.

The value of $l\left(x_{1}, x_{2}, \ldots\right)$ for a specified sequence is generally impossible to determine. Using a devious argument, which will be outlined later, we are able to state the following nonelementary result.

Proposition 2. Let $a$ and $b$ be numbers in the interial [0, 2/3]. For almost all $x$ in $[0,2 / 3], l(x, x, \ldots)=0$.

3. A planar construction. We now do some analogous constructions in the plane. Let $x_{1}$ be a number in $[0,2 / 3]$. By $K^{*}\left(x_{1}\right)$ we denote the set of three closed parallelograms whose vertices in clockwise order are $(0,0)$, $(2 / 3,1),(1,1),(1 / 3,0) ;(2 / 3,0),(0,1),(1 / 3,1),(1,0) ;\left(x_{1}, 0\right),\left(x_{1}, 1\right)$, $\left(x_{1}+1 / 3,1\right),\left(x_{1}+1 / 3,0\right) \cdot T^{*}\left(x_{1}\right)$ will denote the union of these three parallelograms.

If $I$ denotes the unit square $(0,0),(0,1),(1,1),(1,0)$ and $P$ is one of the parallelograms in $K^{*}\left(x_{1}\right)$, then there is a unique affine transformation $\tau$ of the plane which sends the vertices of $I$ to the corresponding vertices of $P$ in the given order. The set $K^{*}\left(x_{1}, x_{2}\right)$ will consist of nine parallelograms of area $1 / 9$ which are the images of the members of $K^{*}\left(x_{2}\right)$ under the three $\tau^{\prime}$ 's associated with $K^{*}\left(x_{1}\right)$.

In general, $K^{*}\left(x_{1}, \ldots, x_{n}\right)$ will consist of $3^{n}$ parallelograms of area $3^{-n}$. For each of the $3^{n-1}$ parallelograms $P$ in $K^{*}\left(x_{1}, \ldots, x_{n-1}\right)$ there is an affine $\tau$ taking $I$ to $P$ with proper vertices corresponding. The set $K^{*}\left(x_{1}, \ldots, x_{n}\right)$ consists of the various images of the members of $K^{*}\left(x_{n}\right)$ under these transformations. We let $T^{*}\left(x_{1}, \ldots, x_{n}\right)$ denote the union of the members of $K^{*}\left(x_{1}, \ldots, x_{n}\right)$ and let $l^{*}\left(x_{1}, \ldots, x_{n}\right)$ denote the planar measure of this union.

If $x_{1}, x_{2}, \ldots$ is a sequence in $[0,2 / 3]$, we define $T^{*}\left(x_{1}, x_{2}, \ldots\right)$ and $l^{*}\left(x_{1}, x_{2}, \ldots\right)$ analogously to their linear counterparts. Also, let $T^{\prime}\left(x_{1}, x_{2}, \ldots\right)$ be the planar set obtained by rotating $T^{* k}\left(x_{1}, x_{2}, \ldots\right)$ through a positive angle of $\pi / 2$ about $(1 / 2,1 / 2)$, the center of $I$.

Lemma 3. If $x_{1}, x_{2}, \ldots$ is any sequence in $[0,2 / 3]$, the planar set $T^{*}\left(x_{1}, x_{2}, \ldots\right) \cup T^{\prime}\left(x_{1}, x_{2}, \ldots\right)$ contains a translate of any line segment lying in the unit square $I$.

Proof. Let $L$ be a line segment in $I$. Let us suppose that the line determined by $L$ and the $x$-axis determine an angle (measured from axis to line) in the interval $[\pi / 4,3 \pi / 4]$. We may assume that $L$ joins a point on the top edge of $I$ to a point on the bottom edge. It is easy to see that at least one $P$ in $K^{*}\left(x_{1}\right)$ contains a translate of $L$. Because affine transformations preserve parallelism, it is seen that $T^{*}\left(x_{1}, \ldots, x_{n}\right)$ will also contain a translate of $L$. By standard arguments, the compact set $T^{*}\left(x_{1}, x_{2}, \ldots\right)$ will contain a translate of $L$. 
If $L$ and the $x$-axis determine an angle in $[-\pi / 4, \pi / 4]$, then $T^{\prime}\left(x_{1}, x_{2}, \ldots\right)$ will contain a translate of $L$. This completes the proof.

Proposition 3. For almost all sequences $x_{1}, x_{2}, \ldots$ in $[0,2 / 3]$ the planar set $T^{*}\left(x_{1}, x_{2}, \ldots\right) \cup T^{\prime}\left(x_{1}, x_{2}, \ldots\right)$ is a B-set.

Proof. For $0 \leq t \leq 1$ let $L_{t}$ be the horizontal line segment joining the points $(0, t)$ and $(1, t)$. We observe that the $y$-section of $T^{*}\left(x_{1}, x_{2}, \ldots\right)$ determined by $L_{t}$ is of the form $T\left(x_{1}, x_{2}, \ldots\right)$ where $a=(2 / 3) t$ and $b=$ $(2 / 3)(1-t)$. The sequence $x_{1}, x_{2}, \ldots$ remains unchanged. Thus for almost all sequences $x_{1}, x_{2}, \ldots$ every $y$-section of $T^{*}\left(x_{1}, x_{2}, \ldots\right)$ has linear measure zero. The result follows at once.

We now state a much deeper result corresponding to Proposition 2.

Proposition 4. If $x$ is any number in $[0,2 / 3]$, except $1 / 3$, then $l^{*}(x, x, \ldots)=0, l^{*}(1 / 3,1 / 3, \ldots)=1 / 2$.

4. Outline of proofs for Propositions 2 and 4. Our next lemma shows that in studying the behaviour of $l(x, x, \ldots)$ we need only consider the case $a=0$ and $b=2 / 3$.

Lemma 4. Let $a, b$ and $x$ be numbers in the interval $[0,2 / 3]$. Then $T(x, x, \ldots)$ is similar to $T\left(x^{\prime}, x^{\prime}, \ldots\right)$ where $a=0$ and $b=2 / 3$.

Proof. We note that the set $T(x, x, \ldots)$ is the union of three similar images of itself, the ratio of similarity being $1 / 3$. Call these images $T_{1}$, $T_{2}$ and $T_{3}$, and let $y_{1}, y_{2}$ and $y_{3}$ be their respective least members. We may assume that $y_{1} \leq y_{2} \leq y_{3}$. If $z$ is the largest member of $T(x, x, \ldots)$, then there is a unique affine transformation $\tau$ such that $\tau\left(y_{1}\right)=0$ and $\tau(z)=1$. It follows that $\tau\left(y_{3}\right)=2 / 3$; we define $x^{\prime}=\tau\left(y_{2}\right)$. It is apparent that $r(T(x, x, \ldots))=T\left(x^{\prime}, x^{\prime}, \ldots\right)$.

If $a \leq x \leq b$, we can see that $y_{1}=3 a / 2, y_{2}=x+y_{1} / 3, y_{3}=b+y_{1} / 3, z=1-$ $3(1-b) / 2$. It is interesting to observe that if $i \neq j, T_{i} \cap T_{j}$ has linear measure zero even though $T(x, x, \ldots)$ may not. From this point on we will always assume that $a=0$ and $b=2 / 3$.

Our proofs of Propositions 2 and 4 depend on the projections of a planar set $E$ which we now define. Let $E_{0}$ be an equilateral triangle of side one. The collection $E_{1}$ will consist of the three homothets of $E_{0}$ of side $1 / 3$ obtained by dilations of ratio $1 / 3$ centered at each of the three vertices of $E_{0}$. We obtain $E_{2}$, a collection of nine equilateral triangles of side $1 / 9$, by performing dilations of ratio $1 / 3$ centered at each vertex of each member of $E_{1}$ so that a member of $E_{1}$ gives rise to three triangles in $E_{2}$. We proceed inductively, and, in general, $E_{n}$ will consist of $3^{n}$ equilateral triangles of side $3^{-n}$. Let $E=\bigcap E_{n}$.

For those familiar with Besicovitch's theory of planar sets of finite 
length, it is easy to see that $E$ is an irregular set of Carathéodory length 1. The fundamental theorem of Besicovitch [2] assures that the almost all directions $\theta$, the linear orthogonal projection $E_{\theta}$ of $E$ possesses zero linear measure.

For each $\theta, E_{\theta}$ is similar to $T(x, x, \ldots)$ for a suitable $x$. In fact, we need only consider certain $\theta$-intervals of length $\pi / 6$ to be assured that for each $x$ a similar image $E_{\theta(x)}$ occurs. Furthermore, it is clear that $x$ and $\theta(x)$ are related in an absolutely bicontinuous manner over any $\theta$-interval in which the mapping $x \rightarrow \theta(x)$ is one to one. It follows that $l(x, x, \ldots)=0$ for almost all $x$ in $[0,2 / 3]$.

Proposition 4 is established in a similar manner by relating the $y$ sections of $T^{*}(x, x, \ldots), 0 \leq y \leq 1$, to $\theta^{*}(y)$ where $E_{\theta^{*}(y)}$ is similar to the $y$-section of $T^{*}(x, x, \ldots)$. This can easily be done in every case, except $x=1 / 3$, to show that $l^{*}(x, x, \ldots)=0$. When $x=1 / 3$, the mapping $y \rightarrow \theta^{*}(y)$ is constant. In fact each $y$-section is similar to $[0,1]$. The set $T^{*}(1 / 3,1 / 3, \ldots)$ consists of the two triangles with vertices $(0,1),(1,1),(1 / 2,1 / 2)$ and $(0,0),(1,0),(1 / 2,1 / 2)$.

We admit that the method of proof outlined above is somewhat artificial and does not readily generalize to constructions involving more than three intervals. We hope that a direct proof of Proposition 2 can be found which will tell precisely for which $x$ it is true that $l(x, x, \ldots)=0$.

\section{REFERENCES}

1. A. S. Besicovitch, On Kakeya's problem and a similar one, Math. Z. 27 (1928), 312-320.

2. - On the fundamental geometrical properties of linearly measurable plane sets of points. III, Math. Ann. 116 (1939), 349-357.

3. - The Kakeya problem, Amer. Math. Monthly 70 (1973), 697-706.

4. William Feller, An introduction to probability theory and its applications. Vol. I, 2nd ed., Wiley, New York, 1957. MR 19, 466. 61801

DEPARTMENT OF MATHEM ATICS, UNIVERSITY OF ILLINOIS, URBANA, ILLINOIS, 